\title{
Amelioration of altered adipokines expression and serine/threonine phosphorylation of IRS1 in alloxan-induced diabetes by Potentilla fulgens and its phytochemical constituents
}

\author{
Shelareen E Sunn, Donkupar Syiem * and Careen Liza Pakyntein \\ Department of Biochemistry, School of Life Sciences, North-Eastern Hill University, Shillong-793022, Meghalaya, India.
}

GSC Biological and Pharmaceutical Sciences, 2021, 17(03), 144-152

Publication history: Received on 13 November 2021; revised on 18 December 2021; accepted on 20 December 2021

Article DOI: https://doi.org/10.30574/gscbps.2021.17.3.0360

\begin{abstract}
The present study is aimed at investigating the modulation of serine/threonine phosphorylation of IRS1 and the gene expression oftotal IRS1 and adipokines including TNF- $\alpha$, IL- 6 and adiponectin by the plant Potentilla fulgens and its phytochemical constituents catechin and (-)-epicatechin. Alloxan-induced diabetic mice with a two-to three-fold increase in their blood glucose levels were taken for the study. The level of protein expression of total (tIRS1), tyrosine (pIRS1), and serine phosphorylated IRS1 (pIRS1 ser307) was analysed by western blot, and the gene expression level of tIRS1, IL-6, TNF- $\alpha$, and adiponectin was analysed by real-time PCR. Since evidences strongly suggest that adiponectin, TNF- $\alpha$, and IL- 6 are implicated in insulin resistance and type 2 diabetes, therefore these three adipokines have been targeted in our study with an aim to investigate the anti-inflammatory and antidiabetic effects of our plant Potentillafulgens(PF) andits phytochemicals. The results strongly demonstrates the capability of PF and its phytochemicals to modulate the ser/thr phosphorylation state of IRS1 by downregulating the serine 307 phosphorylation while simultaneously upregulating the tyrosine phosphorylation of IRS1. The results also indicate the ability of the same to alleviate inflammation in alloxan induced diabetes by modulating the expression of the insulin sensitizing hormone adiponectin and the pro-inflammatory cytokines L- 6 and TNF- $\alpha$.
\end{abstract}

Keywords: Potentilla fulgens; Catechin; (-)-epicatechin; IRS1; Adipokines

\section{Introduction}

In the past, white adipose tissue (WAT) has been regarded as the primary organ for storing and releasing energy in the form of triacylgerol (TAGs) and free fatty acids. However, in the past decade, the identification of "adipokines" as secretory factors from this tissue and their metabolic effects on various tissues indicates a pivotal role that adipose tissue has in whole body glucose, lipid metabolism, and energy homeostasis [1] In both obesity and lipodystrophy, the main function of adipose tissue in maintaining lipid influx is disturbed [2]. Evidence strongly suggests that these adipokines are implicated in the pathophysiology of insulin resistance and are dysregulated in obesity [3, 4].According to Martinez et al. [5], these adipokines are nothing but hormones that play key roles in regulation of insulin sensitivity and energy intake and expenditure. Vendrell [6] suggest that hormones like leptin, adiponectin, resistin, and ghrelin are all involved in the regulation of energy balance and may contribute to promoting the progression of insulin resistance to type 2 diabetes and endothelial dysfunction in the context of increased adiposity. More generally, current studies have revealed that in obese and insulin-resistant subjects, expression and/or secretion of pro-inflammatory adipokines (e.g., TNF, IL-6, resistin, RBP-4, and PAI-1) from white adipose tissue (WAT) is upregulated. Conversely, several novel adipokines (viz., adiponectin, leptin, and omentin) that are positively associated with insulin sensitization are decreased in obesity [7].

\footnotetext{
*Corresponding author: Donkupar Syiem

Department of Biochemistry, School of Life Sciences, North-Eastern Hill University, Shillong-793022, Meghalaya, India
} 
Many, if not all Map kinases (MAPKs) serve as a mechanism for insulin resistance via induction of IRS Ser/Thr phosphorylation and degradation, more specifically, the activation of JNK induces IRS phosphorylation at ser307 and desensitises insulin action in the liver and other tissues [8].Inflammatory cytokines also promote insulin resistance, TNF- $\alpha$ in particular, activates inhibitor $\kappa \beta$ kinase (IKK) which phosphorylates IRS at ser307, prevents binding of IRS to PI3K, inhibits production of adiponectin and thereby promote insulin resistance in liver, muscle and adipose tissue [9]. Potentilla fulgens L. (Rosaceae), a medicinal plant with antioxidant, antihyperglycemic, hypoglycemic, antihyperlipidemic, anti-tumor, anti-inflammatory, and anthelminthic properties [10,11,12, 13, 14]. have been reported to be contain many phytochemicals. However, the phytochemicals catechin and (-)-epicatechin were found to have the highest content $[13,14]$. On this basis, catechin and (-)-epicatechin were selected in this study.

\section{Material and methods}

\subsection{Chemicals and reagents}

(St. Louis, Missouri, USA)Antibodies against IRS1 (ab52167) were procured from Abcam in Cambridge, U.K. The phosphospecific antibodies pIRS1(sc2004) and pIRS1-ser307 (sc33956) were purchased from Santa Cruz Biotechnology, Inc.; ß-actin (sc-81178), anti-rabbit IgG, HRP-linked (sc-2004), anti-mouseIgG, HRP-linked (sc-2005) were obtained from Santa Cruz Biotechnology, Inc. Enhanced chemiluminescence (ECL) western blotting detecting reagent (code no. 170-5061), precision plus protein unstained standards (cat \# 161-0362), and precision protein StrepTactin-HRP conjugate (cat \# 161-0380) were purchased from Biorad. Sybr Green was purchased from Biorad. Primers were purchased from Integrated DNA Technologies Inc. Other reagents and chemicals were supplied by Sisco Research Laboratories (SRL), India and were of the highest analytical grade.

\subsection{Methods}

\subsubsection{Experimental animals}

Healthy Swiss albino mice (BALB/c strain) weighing 25-30g, procured from the Pasteur Institute, Shillong, Meghalaya, were used for the study after approval from the Institutional Ethical Committee (IEC: Animal model/NEHU 15-122015).

\subsubsection{Plant Extraction}

The roots of PF were washed, dried, and ground into powdered form. One half of the dried powdered form was repeatedly extracted with 10 volumes of aqueous-methanol solution in a ratio of 1:4 [15].The mixture was filtered and the filtrate was dried under vacuum in a rotary evaporator (Yamato RE800), yielding the crude methanolic extract.

\subsubsection{Preparation of diabetic mice}

Animals were given a single dose intravenous (i.v) administration of alloxan monohydrate (80 mg/kg b.w.) [16] prepared in acetate buffer $(0.15 \mathrm{M}, \mathrm{pH} 4.5)$ and fasting blood glucose levels were determined 72 hours post administration. The control group received only the buffer. Prior to administration, mice were fasted overnight but given water ad libitum. Mice with blood glucose level $\geq 200 \mathrm{mg} / \mathrm{dL}$ were considered diabetic and used for the study[10].

\subsubsection{Sub-acute studies}

Mice were divided into eight groups. Treatment was done alternately for a period of 14 days after which the animals were deprived of food overnight and sacrificed by cervical dislocation. Epididymal adipose tissues were excised, weighed and used for western blot analysis and real-time PCR. Both Cat and EC at a dose of $10 \mathrm{mg} / \mathrm{kg}$ b.w was able to decrease fasting blood glucose at same significance level as $20 \mathrm{mg} / \mathrm{kg}$ b.w in the 24 hours antihyperglycemic study (data not shown).Therefore $10 \mathrm{mg} / \mathrm{kg}$ b.w was taken as the optimum dose and used for the study.

- $\quad$ Group 1: normal mice treated with vehicle alone (2\% ethanol).

- Group 2: alloxan-induced diabetic mice.

- Group 3: alloxan-induced diabetic mice treated with the effective optimum dose of Cat $10 \mathrm{mg} / \mathrm{kg}$ b.w., respectively.

- Groups 4: alloxan-induced diabetic mice treated with the effective optimum dose of EC $10 \mathrm{mg} / \mathrm{kg}$ b.w respectively. 
- Group 5: alloxan-induced diabetic mice treated with a combination of Cat and EC $(10 \mathrm{mg} / \mathrm{kg}$ b.w dose in a 1:1 ratio).

- Group 6: alloxan-induced diabetic mice treated with a combination of Cat, EC and piperine (10 mg/kg b.w dose in a $1: 1: 1$ ratio).

- Group 7: alloxan-induced diabetic mice treated with PF (250 mg/kg b.w).

- Group8: alloxan-induced diabetic mice treated with Met, (500 mg/kg b.w) respectively [17].

\subsubsection{Protein extraction for epididymal adipose tissue}

The method of Twinn [18], with slight modifications was followed to extract IRS1 and AKT proteins from the epididymal adipose tissues. In brief, the tissues were lysed in lysis buffer (containing 50mM/L HEPES, pH 8.0, $150 \mathrm{mM} \mathrm{NaCl}, 1 \%$ Triton $\mathrm{X}, 30 \mathrm{mM} \mathrm{NaF}, 1 \mathrm{mM} \mathrm{Na} 3 \mathrm{VO}_{4}, 10 \mathrm{mM} \mathrm{Na} 4 \mathrm{P}_{2} \mathrm{O}_{7}, 10 \mathrm{mM}$ EDTA, $1 \mu \mathrm{g} / \mathrm{ml}$ leupeptin, $1 \mu \mathrm{g} / \mathrm{ml}$ aprotinin).Protease inhibitor cocktail was added fresh to the buffer. Homogenates were kept on ice for $20 \mathrm{~min}-1 \mathrm{~h}$ and then centrifuged at $12000 \mathrm{rpm}$ for $40 \mathrm{~min}$ at $4^{\circ} \mathrm{C}$. Protein concentration was determined using Bradford reagent [19]. Protease inhibitor cocktail was added fresh to the buffer. . Equal amounts of protein samples were separated by 7.5\% SDS PAGE and transferred to nitrocellulose membranes using Mini Trans-blot Turbo Transfer system (Bio-Rad, USA). Membranes were probed with the corresponding primary antibodies IRS1 and ser 307 pIRS1 overnight at $4^{\circ} \mathrm{C}$.The secondary antibody used was goat anti-mouse secondary antibody. The protein was visualised using enhanced chemiluminescence substrate and analysed with the Chemi Doc MP imaging system Bio-Rad. $\beta$-actin was used as the loading control. For the detection of tyrosine phosphorylated IRS1, membrane used for detection of total IRS1 was marked at the site of IRS1 protein, stripped and probed with phosphospecific antibody.

\subsubsection{RNA Isolation and real time PCR}

Total RNA was isolated from epididymal adipose tissues by using combined methods of TRI reagent plus RNeasy method (RNeasy Plus fibrous mini kit, Qiagen). cDNA synthesis from $1 \mu \mathrm{g}$ pure RNA was carried out using iScript cDNA synthesis kit from Biorad. The resultant cDNA samples were processed for real time PCR using specific primers for total IL-6, TNF$\alpha$, Adiponectin and GAPDH in a reaction mixture of a total volume of $10 \mu \mathrm{l}$.

\subsection{7. cDNA Synthesis}

cDNA synthesis from $1 \mu \mathrm{g}$ pure RNA was carried out using iScript cDNA synthesis kit from Biorad. The reaction mix was incubated for $5 \mathrm{~min}$ at $25^{\circ} \mathrm{C}$, followed by $30 \mathrm{~min}$ at $42^{\circ} \mathrm{C}$ to activate reverse transcription. The reverse transcriptase enzyme was then inactivated by incubation at $85^{\circ} \mathrm{C}$ for $5 \mathrm{~min}$ and hold at $4{ }^{\circ} \mathrm{C}$. The resultant cDNA samples were processed for real time PCR using specific primers for the target proteins and GAPDH in a reaction mixture of a total volume of $10 \mu$ l.The cDNA was stored at -80 until use.

\subsubsection{Primer designing}

The nucleotide primers were designed using the Primer3 algorithm of the Primer-Blast software from the National Centre for Biotechnology Information (NCBI) (www.ncbi.nlm.nih.gov/tools/primerblast/index.cgi?LINK_LOC=BlastHomeAd).The following primers were used:

\section{IL-6-Forward 5' gacaaagccagagtccttcaga 3'; Reverse 5' TGTGACTCCAGCTTATCTCTTGG 3'}

TNF-Forward 5' gtgaccactctgccgtgtat 3'; Reverse 5' tattgttgctgctggagagcc 3'

Adiponectin-Forward 5' tgacgacaccaaaagggctc 3' 5 cacaagttccctttgggtgga $3^{\prime}$

\section{GAPDH-Forward $5^{\prime}$ aggtcggtgtgaacggatttg $3^{\prime}$ Reverse $5^{\prime}$ tgtagaccatgtagttgaggtca $3^{\prime}$}

Quantitative expression of genes of interest (i.e. IL-6, TNF- $\alpha$, adiponectin) was determined and carried out on a CFX96 ${ }^{\mathrm{TM}}$ real-time PCR detection system which comprises of C1000 ${ }^{\mathrm{TM}}$ thermal cycler, CFZ96 optical reaction module and operated by CFX Manager ${ }^{\mathrm{TM}}$ software. Human GAPDH was used as an endogenous reference gene. A real time PCR reaction in a $10 \mu$ final volume was performed using the mastermix Dynamo SYBR Green supermix from Biorad. Real time data was calculated using double delta $\mathrm{Ct}$ analysis ( $\Delta \Delta \mathrm{ct}$ relative quantification method) given by Livak [20] and the data were normalized to a human GAPDH which was used as the endogenous reference control. 


\section{Results and discussion}

\subsection{Effect of PF, catechin and epicatechin, and theircombination on the expression of total IRS1, tyrosine and serine 307 phosphorylated IRS1 (t-IRS1, p-IRS1 and ser 307 p-IRS1)}

When compared to the control group, the total IRS1 protein level in DC was reduced by $83 \%$ ( $<<0.001)$. Results revealed that the t-IRS1protein of all treated groups was significantly increased to a similar extent (Fig 1B). The t-IRS1 mRNA level in the epididymal adipose tissue of diabetic mice was considerably reduced to $\sim 0.1$ fold. This reduction was remarkably increased in all the treated groups. The t-IRS1 mRNA levels were altered by a fold change of $\sim 0.3$ (p<0.01) , 0.9 (p<0.05), 1.3 ( $\mathrm{p}<0.001), \sim 1(\mathrm{p}<0.05)$, and 1.25 ( $\mathrm{p}<0.001)$ for diabetic groups treated with Cat, Cat/EC, Cat/ECP, PF and Met respectively (Fig 1E).The expression of tyrosine $p$-IRS1 protein decreased significantly by $84 \%(p<0.001)$ in the skeletal muscle of DC as compared to NC. This reduction was significantly elevated in all the treated groups. (Fig 1 C) On the other hand, the p-IRS1 (ser 307) protein which got significantly elevated in the diabetic control was significantly lowered in the treated groups (Fig 1 D). Maximum reduction was seen in the extract treated group decreasing the level to $93 \%(\mathrm{p}<0.001)$ respectively.

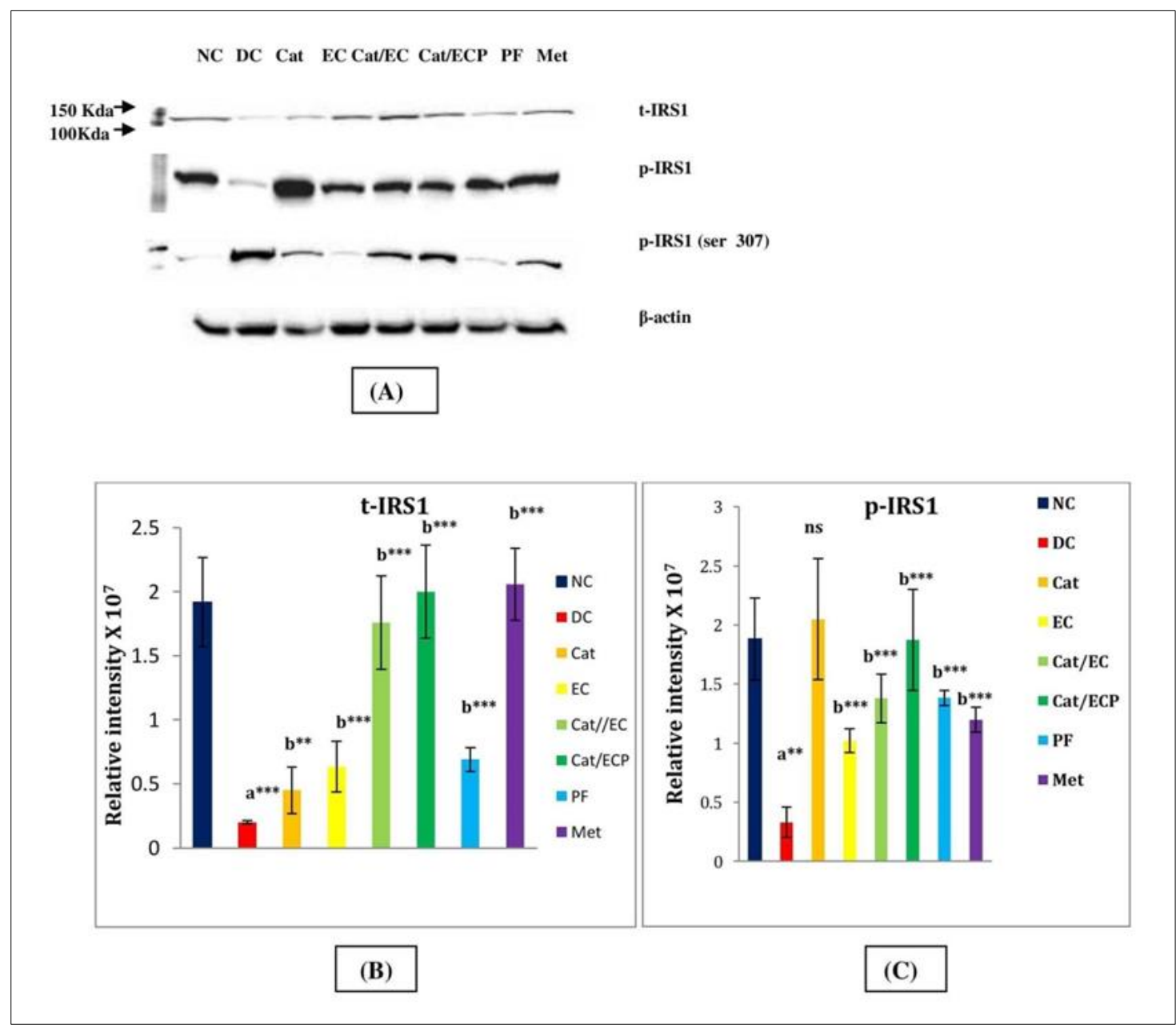




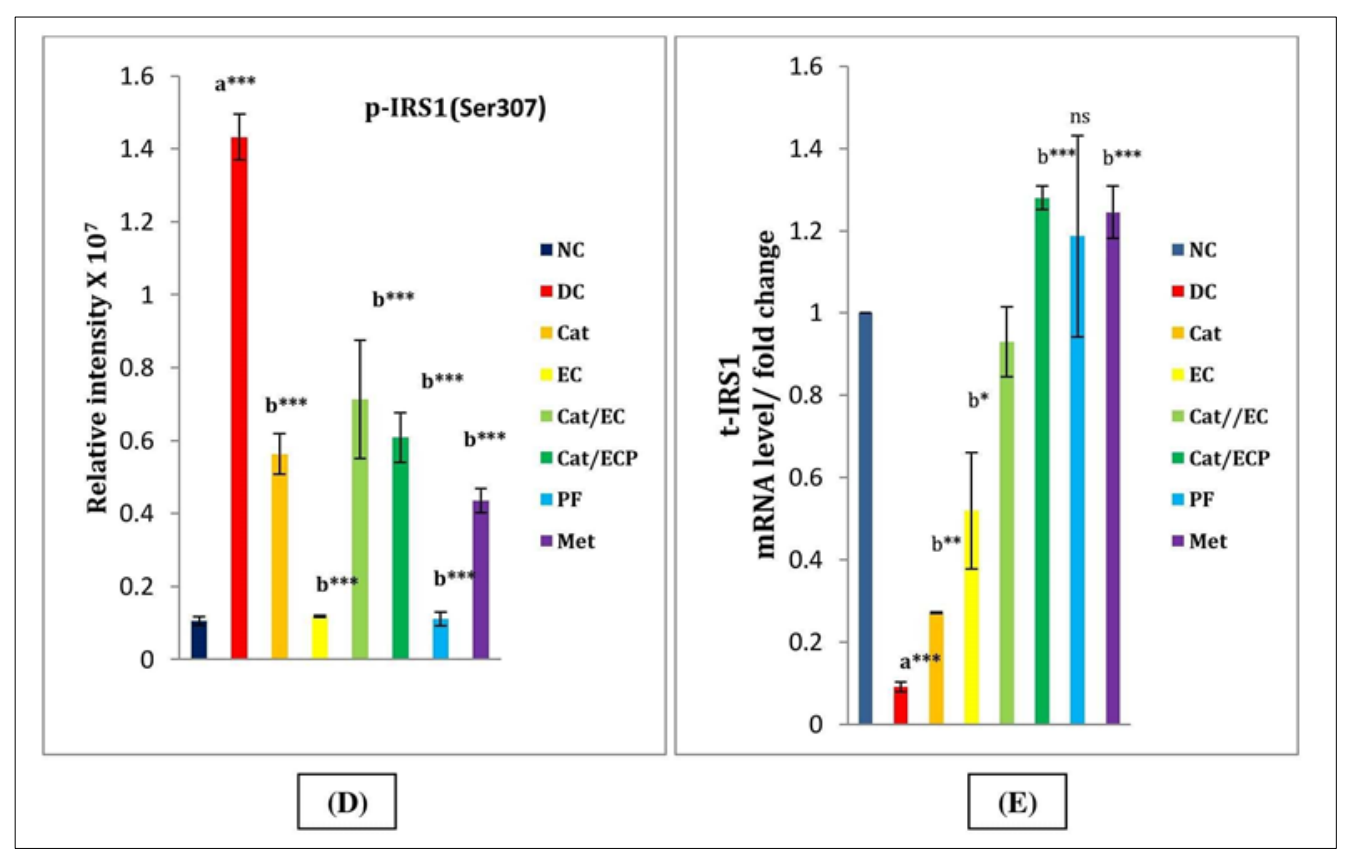

Figure 1(A) Representative western blot and corresponding densitometric analysisof (B) t- IRS1, (C) p-IRS1, (D) p-IRS1 (ser307) with $\beta$-actin was as the loading control. (E) Expression level of t-IRS1 mRNA proteins in the epididymal adipose tissues of NC, DC, Cat, EC, Cat/EC, Cat/ECP, PF, and Met-treated mice normalized to GAPDH. Fold change of diabetic mice was calculated and compared against normal mice and those of treated diabetic mice were calculated in comparison to diabetic mice using the $2 \Delta \Delta \mathrm{ct}$ method. Values are expressed as mean \pm SEM of triplicates, p-represents level of significance at ${ }^{* * *} \mathrm{p}<0.001,{ }^{* *} \mathrm{p}<0.01$ and ${ }^{*} \mathrm{p}<0.05$ respectively (a denotes level of significance against NC; ${ }^{b}$ denotes level of significance against DC. (NC- normal, DC- diabetic control, Cat - catechin hydrate, EC: (-)-epicatechin, Cat/EC- catechin hydrate and (-)-epicatechin, Cat/EC/P- catechin hydrate, (-)-epicatechin and piperine, PF- potentilla fulgens, MetMetformin. a-represents level of significance of DC against NC, b- represents level of significance of DC against treated groups.ns-non-significant).

\subsection{Effect of PF, and catechin, epicatechin, and their combination on adiponectin gene expression in epidydimal adipose tissue}

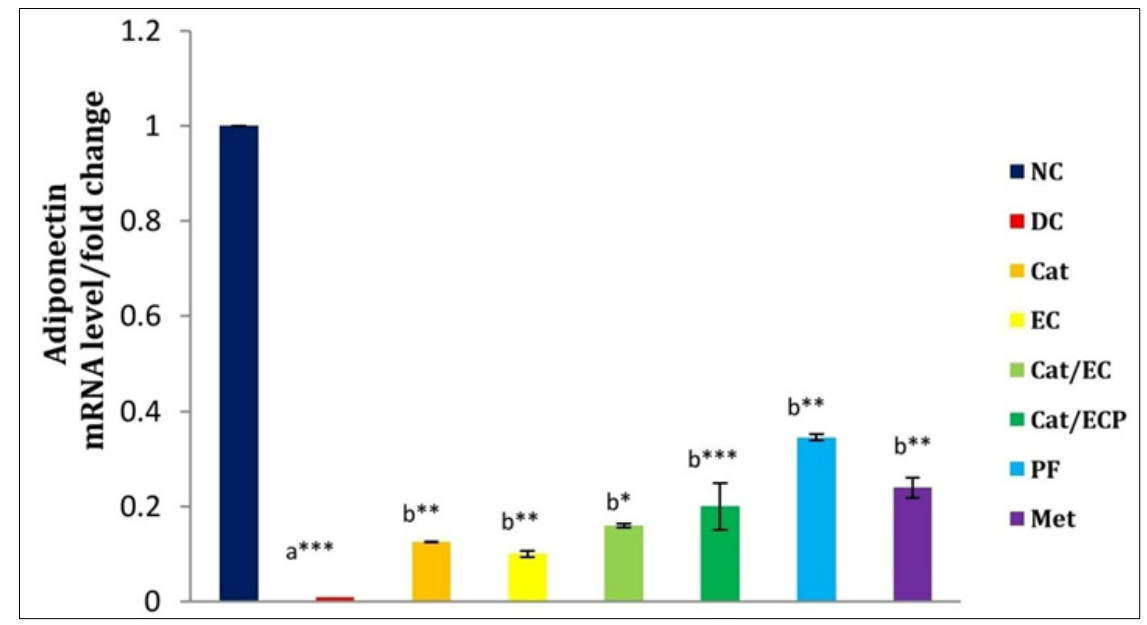

Figure 2 Adiponectin mRNA expression in the epididymal adipose tissue of NC, DC, Cat/EC, Cat/ECP, PF, and Mettreated mice were normalized to GAPDH. Statistical significance compared to respective controls is denoted by ' $\mathrm{a}$ ' when compared against NC and ' $b$ ' when compared against DC. Level of significance was fixed at ${ }^{*} p<0.05,{ }^{* *} p<0.01$ and ${ }^{* * *} \mathrm{p}<0.001$ respectively. The fold change of diabetic mice was calculated and compared against normal mice, and those of treated diabetic mice were calculated in comparison to diabetic mice using the $2^{\Delta \Delta c t}$ method 
The mRNA level of adiponectin was considerably downregulated to $\sim 0.06$ fold $(\mathrm{p}<0.001)$ in the diabetic state as compared to NC (Fig 2). Results showed that the mRNA expression of this adipokine was significantly upregulated in the group treated with the extract, where the fold change was $\sim 0.35(\mathrm{p}<0.001)$ from the diabetic control. As shown in the figure, the standard drug metformin could increase the adipokine's expression and upregulate its expression to $\sim 0.24$ fold $(\mathrm{p}<0.05)$ from the diabetic control. Both Cat/EC and Cat/ECP had similar effects on upregulating the adiponectin mRNA levels where the relative fold change of increase from DC were $\sim 0.18(\mathrm{p}<0.001)$ and $\sim 0.16$ folds $(\mathrm{p}<0.05)$ respectively.Catechin also displayed a significant effect in upregulating the expression of adiponectin, increasing it to $\sim 0.12$ fold $(\mathrm{p}<0.01)$ respectively.

\subsection{Effect of PF, catechin and epicatechin, and their combination on TNF-gene expression in epididymal adipose tissue}

The mRNA level of the inflammatory cytokine, Tumor Necrosis Factor alpha (TNF- $\alpha$ ), was significantly increased to $\sim 16$ fold $(\mathrm{p}<0.001)$ in the diabetic state as compared to NC (Fig 3). Results show that this reduction was significantly increased in all the treated groups. As shown in the figure, Cat/ECP, Met and PF were more effective in reducing the cytokine's expression, decreasing the cytokine's expression by $\sim 0.5(\mathrm{p}<0.01), \sim 0.6(\mathrm{p}<0.001)$ and $\sim 1.3$ ( $\mathrm{p}<0.001)$ folds, respectively, from the diabetic control. Both Cat and EC reduced TNF levels by $\sim 2.4(\mathrm{p}<0.001)$ and $\sim 2.5$ ( $\mathrm{p}<0.001)$ folds, respectively, when compared to the diabetic control. The combined group, Cat/ECP and Met, had similar effects on TNF expression levels where the relative fold change in decreasing the cytokine's expression was $\sim 0.5(\mathrm{p}<0.01)$ and $\sim 0.6$ folds $(\mathrm{p}<0.001)$, respectively, when compared against DC

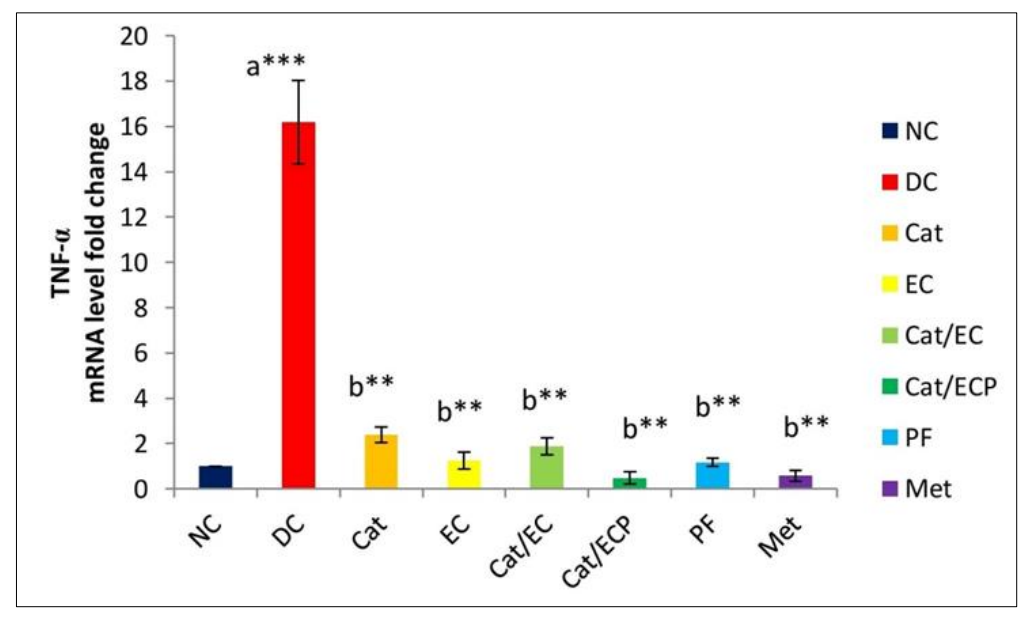

Figure 3 TNF- $\alpha$ mRNA expression in epididymal adipose tissue of NC, DC, Cat, EC, Cat/ECP, PF, and Met-treated mice was normalized to GAPDH. Statistical significance compared to respective controls is denoted by ' $\mathrm{a}$ ' when compared against NC and 'b' when compared against DC. Level of significance was fixed at $* \mathrm{p}<0.05,{ }^{* *} \mathrm{p}<0.01$ and ${ }^{* * *} \mathrm{p}<0.001$ respectively. The fold change of diabetic mice was calculated and compared against normal mice, and those of treated diabetic mice were calculated in comparison to diabetic mice using $2 \Delta \Delta \mathrm{ct}$ method

\subsection{Effect of PF, catechin and epicatechin, and their combination on IL-6 gene expression in epididymal adipose tissue}

The gene expression of the cytokine Interleukkin-6 (IL-6) was considerably increased to $\sim 8.7$ fold ( $p<0.01$ ) in the diabetic control as compared to NC (Fig 4). Results indicate that this upregulation was significantly decreased in all the treated groups. As shown in the figure, Met and PF were the most effective in reducing the cytokine's expression, decreasing its mRNA level to $\sim 0.6$ ( p 0.01) and $\sim 0.8(\mathrm{p}<0.01)$ folds from the diabetic control, respectively. Cat/ECP could reduce the IL- 6 expression level by $\sim 2.5$ folds $(\mathrm{p}<0.05)$ compared to the diabetic control. The relative fold change in decreasing the cytokine'sexpression were $\sim 4(\mathrm{p}<0.01)$ and $\sim 1$ folds $(\mathrm{p}<0.01)$ for Cat and EC when compared against DC while the combined group Cat/EC could reduce the cytokine's expressionto $\sim 3$ fold but the result was non-significant against DC. 


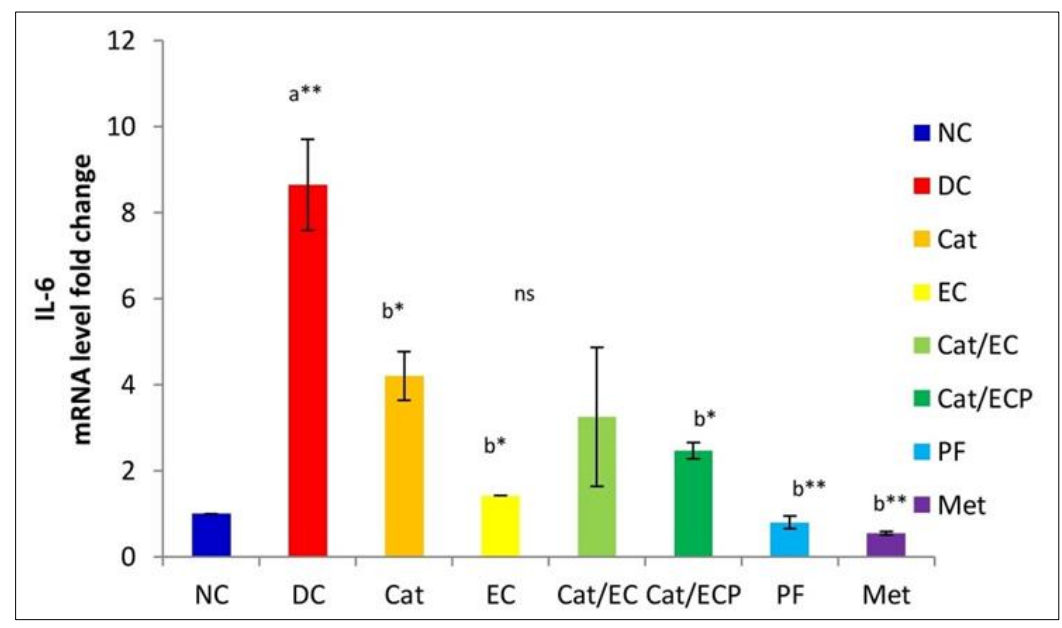

Figure 4 IL-6 mRNA in epididymal adipose tissue of NC, DC, Cat/EC, Cat/ECP, PF and Met-treated mice was normalized to GAPDH. Statistical significance compared to respective controls are denoted by ' $a$ ' when compared against NC and ' $b$ ' when compared against DC. Level of significance was fixed at $* p<0.05,{ }^{* *} p<0.01$ and ${ }^{* * *} \mathrm{p}<0.001$ respectively. Fold change of diabetic mice was calculated and compared against normal mice and those of treated diabetic mice were calculated in comparison to diabetic mice using the $2^{\Delta \Delta c t}$ method

\section{Discussion}

In this study, the effect of PF and its phytochemical constituents, on the tyrosine and serine 307 phosphorylation of IRS1 in alloxan diabetic mice was assessed. The total protein and gene expression of IRS1 was also evaluated. Simultaneously, the adipokines expression in the epididymal adipose tissues of these mice were also evaluated with an aim to correlate the adipokines mediated inflammation on insulin signaling. It is currently well established that tyrosine phosphorylation causes activation while Ser/Thr phoshorylation of insulin receptor substrate (IRS) proteins causes inhibition of insulin signaling [8]. One of the pathways by which this inhibitory phosphorylation is initiated is through inflammatory cytokines [21], which include TNF- $\alpha$, IL-6, and IL-1 $\beta$ that are secreted in the macrophages and act in a paracrine manner to activate the stress/serine kinases (including IkB kinase $\beta$ (IKK $\beta$ ), c-Jun N-terminal kinase (JNK), S6K and mTOR) in the adipocytes [22]. These kinases phosphorylate at Ser/Thr residues of IRS-1 thus causing insulin resistance in adipocytes [8]. For example, the activation of IKK $\beta$ can impair insulin signaling either directly by phosphorylating IRS-1 on serine residues or through production of multiple inflammatory cytokines, including TNF- $\alpha$ and IL-6 via the transcription factor NFKB [23]. In their review, Wellen and Hotamisligil stated the facts and evidences that strongly suggest that diabetes is an inflammatory disease with inflammation as the primary cause rather than a mere consequence of obesity-related insulin resistance, hyperglycemia, and hyperlipidemia [23].Our findings showed the over-expression of inflammatory cytokines which further aggravates hyperglycemia, and inhibition of tyrosine phosphorylation of IRS-1 proteins in the adipose tissues of alloxan mice and the plant extract as well as the phytochemicals could alleviate this condition by modulation of the adipokine expression as well as the IRS-1 phosphorylation. Therefore our findings are also consistent with the reviews of Wellen and Hotamisligil which show the presence of inflammation in diabetes.

\section{Conclusion}

Current findings provide insights into the mechanism of anti-inflammatory action of the plant PF conferred upon by the presence of the phytochemicals catechin and epicatechin respectively. Our study also demonstrates the capability of PF and its phytochemicals to modulate the ser/thr phosphorylation state of IRS1. Therefore, this plant could likely avert inflammatory stress-induced complications associated with diabetes and improve insulin signaling in alloxan-induced diabetes.

\section{Compliance with ethical standards}

\section{Acknowledgments}

We thank the UGC for sponsoring the University with Potential for Excellence Project and Department of Biochemistry, North-Eastern Hill University, Shillong, India for providing facilities. 


\section{Disclosure of conflict of interest}

The authors declare no conflict of interest.

\section{Statement of ethical approval}

The study has been performed in accordance with the Institutional Ethics Committee (Animal models) dated $15^{\text {th }}$ December, 2015.

\section{References}

[1] Holst D, Grimaldi PA. New factors in the regulation of adipose differentiation and metabolism. Current Opinion in Lipidology. 2002; 13: 241-245.

[2] Frayn K. N. Adipose tissue as a buffer for daily lipid influx. Diabetologia.2002; 45(9): 1201-10.

[3] Qatanani M and Lazar M A. Mechanisms of obesity-associated insulin resistance: many choices on the menu. Genes\&Development. 2007; 21:1443-1455.

[4] Kwon H and Pessin J. E. Adipokines mediate inflammation and insulin resistance. Frontiers in Endocrinology. 2013; 12(4): 71.

[5] Martinez C. K., Stiles B, Wang Y et al., Insulin Hypersensitivity and Resistance to Streptozotocin-Induced Diabetes in Mice Lacking PTEN in Adipose Tissue. Molecular and Cellular Biology. 2005; 25(6): 2498-2510

[6] Vendrell J, Broch M, Vilarrasa N,MolinaA et al., Resistin, Adiponectin, Ghrelin, Leptin, and Proinflammatory Cytokines: Relationships in Obesity. Obesity Research 2004; 12(6): 962-971.

[7] Makki K, Froguel P, and Wolowczuk Isabelle. Adipose Tissue in Obesity-Related Inflammation and Insulin Resistance: Cells, Cytokines, and Chemokines. ISRN Inflammation. 2013; 5091: 139239.

[8] Haeusler RA, Mc Graw TE, Accili D. Biochemical and cellular properties of insulin receptor signaling. Nature ReviewsMolecular Cell Biology. 2018; 19(1): 31-44.

[9] Ruan H, Lodish HF. Insulin resistance in adipose tissue: direct and indirect effects of tumor necrosis factor-alpha. Cytokine and Growth Factor Reviews. 2003; 14(5): 447-55.

[10] Syiem D, Syngai G, Khup PZ, Khongwir BS, Kharbuli B and Kayang H, Hypoglycemic effects of Potentilla fulgens L. in normal and alloxan induced diabetic mice.Journal of Etnopharmacology. 2002; 83: 55-61.

[11] Syiem D, Syngai G, Kharbuli B, et al., Anti-tumor activity of crude root extract of Potentilla fulgens. Indian Drugs. 2003; 40(2).

[12] Roy B, Swargiary A, Syiem D, Tandon V. Potentilla fulgens (Family Rosaceae), a medicinal plant of north-east India: a natural anthelminthic? Journal of Parasitic Diseases. 2010; 34(2): 83-8.

[13] Saio V, Syiem D, Sharma R, Dkhar J. Amelioration of age-dependent increase in oxidative stress markers in male mice by extract of Potentilla fulgens.Redox Report. 2016; 21: 130-8.

[14] Anal JMH, Majeed AR, Bez1 G, et al., AK In vitro cytotoxicity of the polar extracts of Potentilla fulgens L. against human cancer cell lines: Detection and isolation of bioactive phenolics. Journal of Chemical and Pharmaceutical Research. 2014; 6(9): 89-95.

[15] Harborne JB. Photochemical Methods, $3^{\text {rd }}$ edition. 1998.

[16] Thabah D, Syiem D, Pakyntein CL, et al. A. Potentillafulgens upregulate GLUT4, AMPK, AKT, and insulin in alloxaninduced diabetic mice: an in vivo and in silico study. Archives in physiology and Biochemistry. 2021; 18: 1-13.

[17] Meng XM, Ma XX, Tian YL,Jiang Q, Wang LL, Shi R,Ding L,Pang SG.Metformin improves the glucose and lipid metabolism via influencing the level of serum total bile acids in rats with streptozotocin-induced type 2 diabetes mellitus. European Review for Medical and Pharmacological Sciences. 2017; 21: 2232-2237.

[18] Twinn DSF, Alfaradhi MZ, Gronert MSM, et al., Downregulation of IRS-1 in adipose tissue of offspring of obese mice is programmed cell-autonomously through post-transcriptional mechanisms. Molecular Metabolism. 2014; 20: 3(3):325-33.

[19] Bradford MM.A rapid and sensitive Method for the Quantification of Microgram Quantities of Protein Utilizing the Principle of Protein-Dye Binding.Analytical Biochemistry. 1976; 72: 248- 254. 
[20] Livak KJ, Schmittgen TD. Analysis of relative gene expression data using real-time quantitative PCR and the 2(delta delta C (T)) method. Methods (San Diego, Calif.). 2002; 25(4):402-408.

[21] Hotamisligil GS, Shargill NS, Spiegelman BM. adipose expression of tumor necrosis factor-alpha:direct role in obesity-linked insulin resistance.Science. 1993; 259: 87-91.

[22] Osborn 0, Olefskky JM. The cellular and signaling networks linking the immune system and metabolism in disease. Nature Medicine. 2012; 18:363-374.

[23] Wellen KE, Hotamisligil GS. Inflammation, stress and diabetes.Journal ofClinical Investigation. 2005; 115: 11111119. 